\title{
Knowledge and attitude toward hearing impairment and vestibular disorders among allied health professionals in Delhi NCR
}

\author{
Himanshu Kumar Sanju ${ }^{1 *}$, Komal Aggarwal ${ }^{2}$, Arun Kumar Yadav ${ }^{3}$ \\ ${ }^{\mathbf{1}}$ Assistant Professor, ${ }^{\mathbf{2}}$ Audiologist, ${ }^{\mathbf{3}}$ Assistant Professor, ${ }^{\mathbf{1} D e p t . ~ o f ~ E N T ~ a n d ~ A u d i o l o g y, ~}{ }^{\mathbf{2} 3}$ Dept. of Audiology, ${ }^{\mathbf{1}}$ Shri Jagdamba Charitable \\ Eye Hospital, Sri Ganganagar, Rajasthan, ${ }^{1}$ LKC Shri Jagdamba Andh Vidyalaya Samiti, Sri Ganganagar, Rajasthan, 2,3 Amity Medical \\ School, Amity University, Haryana, India
}

*Corresponding Author: Himanshu Kumar Sanju

Email: himanshusanjuaslp@gmail.com

\begin{abstract}
Numerous studies have concluded that for successful implementation of hearing and speech related programs in a multidisciplinary health care-based set-up, allied health care professionals specialized in any field should have appropriate knowledge and attitude towards the challenges and impact of hearing loss. So, in order to provide abundant knowledge to the allied health professionals audiologists should have evidence based knowledge which can reflect them the actual manifestation regarding the knowledge and attitude of the specialized allied health professionals in areas related hearing loss, assessment, diagnosis and subsequent audiological management. The present research is being conducted to assess the knowledge and attitude among allied health professionals across various range of hospital set up in northern India regarding hearing loss, assessment procedures and intervention options available. A questionnaire-based study (online survey) of 22 questions was adopted from similar kind of studies and was framed with the aim to assess the knowledge and attitude among allied health professionals regrading hearing loss and vestibular disorder. A total of 160 health professionals consisting of psychologist, physiotherapist, optometrist, occupational therapist recruited from medical colleges, government hospitals, primary health centers, ambulatory health care setting participated in the present study. The outcome of the present study reveals that participants had good knowledge and attitude towards the basic areas related to hearing loss and vestibular disorder. However, there are gaps in certain areas where there is a need to increase awareness and educate them regarding the role of an audiologist/speech language pathologist.
\end{abstract}

Keywords: Knowledge, Attitude, Hearing Impairment, Vestibular disorder, Allied health professionals.

\section{Introduction}

In September 2012 the organization of International Chief Health Professions Officers (ICHPO) ${ }^{1}$ provided an agreedupon definition of an allied health professional. 'Allied Health Professions are a distinct group of health professionals who apply their expertise to prevent disease transmission, diagnose, treat and rehabilitate people of all ages and all specialties. Together with a range of technical and support staff they may deliver direct patient care, rehabilitation, treatment, diagnostics and health improvement interventions to restore and maintain optimal physical, sensory, psychological, cognitive and social functions.' Allied health professionals works in hospitals, ambulatory health care setting, nursing and residential care facilities. According to a study being conducted by Varshney (2016), in India 4 in every 1000 children suffer from severe to profound hearing loss. ${ }^{2}$ The impact of delayed diagnosis and proper intervention in infants with hearing loss has a severe negative effect on the overall speech and language, as well as the cognitive and social skills. ${ }^{3,4}$ However there are studies being conducted which states that these negative consequences can be reduced to some extent or prevented through early identification and intervention. ${ }^{5,6}$ Allied health professional working with general practitioners, otorhinolaryngologists (ENTs), pediatricians, neurologist, obstetricians/gynecologists, speech language pathologist and audiologists, are often involved together in the hearing testing of newborns and children. ${ }^{7}$ Allied health professionals frequently work together in various setups such as government and corporate hospitals, primary health centers, medical college, camps, clinics, and private institutes. Allied health professional may play an important role in guiding patients and their families through diagnostic and treatment procedures. Similarly, allied health professional should also be aware about vestibular disorders as patients with vestibular disorders visits neurologist, otolaryngologist, general physician and audiologist. Knowledge about vestibular disorders helps the allied health professionals in guiding patients about evaluation and management by concerned professionals. ${ }^{8}$

Patients with hearing and vestibular disorders often visit medical colleges, government hospitals, primary health centers, ambulatory health care setting, nursing, rehabilitation and residential care facilities. Allied health professionals may play an important role in guiding patients and their families through diagnostic and treatment procedures of hearing and balance disorders. Yerraguntla et al. in $2016^{9}$ investigated knowledge and attitude of medical intern in coastal Karnataka, showed positive attitude toward different hearing related issues. There is a dearth of literature regarding attitude and knowledge about hearing impairment and balance disorders among allied health professional of Delhi NCR. The present study aims to investigate knowledge and attitude toward hearing and balance disorders among allied health professionals.

\section{Materials and Methods Participants}

A total of 160 allied health professionals (psychologist, physiotherapist, optometrist, occupational therapist) recruited from medical colleges, government hospitals, primary health centers, ambulatory health care setting, 
nursing, rehabilitation and residential care facilities. Informed consent were taken from all participants. All the participants were aware about the purpose of study. All the participants were native speaker of Hindi having good knowledge of English.

\section{Questionnaire}

A questionnaire-based study (online survey) of 22 questions was designed and adopted from similar studies to assess the knowledge and attitude towards hearing impairment and vestibular disorders among allied health professionals. ${ }^{9-11}$ The questions were closed set task with 5 point rating scale from strongly agree to strongly disagree (Strongly agree, agree, neutral, disagree and strongly disagree). The questions used to assess knowledge and attitude towards hearing impairments includes 'Hearing loss is a condition that can become extremely disabling', 'hearing aid can be fitted at any age', 'importance of speech language therapy for children using hearing aids', 'learning to read and to write depends more on hearing than sight', 'hearing is an important sense in the development of speech and language of the child', 'importance of new born hearing screening', 'reservation in job and education for individuals with hearing impairment', 'hearing and speech developmental milestone play significant role in child's overall development', 'effect of hearing and speech disorder on social life of the patient', 'listening music at higher volume can cause hearing', 'Consanguinity (marriage in blood relation) can cause hearing loss', 'if a person is feeling ringing/buzzing sound in the ear, which professional he/she should contact'. Few question with 2 points rating scale includes 'Is it possible for young people like you to lose their hearing' and. Have you ever heard the course like Speech and Hearing or Audiology and Speech Language Pathology. A open set question 'Which professional are responsible for hearing assessment?' was also asked from allied health professionals.

The questions used to assess knowledge and attitude towards vestibular disorders includes 'balance problem could be due to dysfunction in his/her inner ear', 'vertigo can occur with tinnitus', 'in case of vertigo, which professional to contact'.

\section{Data Analysis}

Data collected was statistically calculated using SPSS 20 (SPSS Inc., South Asian Ed, Bengaluru, Karnataka, India). Descriptive statistics was used to summarize the data and the scores thus, obtained were cross-tabulated to obtain percentages.

\section{Result}

The percentages of the responses being obtained in the different categories of questionnaires have been described under different headings which is based on the rating scale through graphical representation. The data obtained was analyzed and percentage of responses were calculated.
Knowledge and attitude towards awareness, services and referrals towards individuals with hearing impairment

A set of positive and negative statements were framed in the questionnaire in order to avoid repetition of the responses regarding all questions being asked.70\% of the allied health professionals agreed the fact that hearing loss can become extremely disabling as shown in Figure $1.60 \%$ of the allied health professional believes that hearing assessment tests are not invasive and can be performed at any age, as shown in Fig. 2. Only $56 \%$ of the allied health professional believe that in case of hearing impairment, hearing aid can be fitted at any age. Only $65 \%$ of the allied health professionals agreed the importance of speech language therapy and listening training for children with hearing impairment. Only $44 \%$ of the allied health professionals supported the notion that learning to read and to write depends more on hearing than sight. $99 \%$ of the allied health professionals believes the fact that hearing is an important sense in the development of speech and language of the child. Figure 3 shows that $74 \%$ of the allied health professionals agreed the importance of newborn hearing screening and again $74 \%$ of the allied health professional supported reservation in jobs and education of individuals with hearing impairment. $96 \%$ of the allied health professionals supported the notion that hearing and speech developmental milestone play significant role in child's overall development. $78 \%$ of the allied health professional believes that communication disorders have a negative effect on social and professional life of the individual. $53 \%$ of the allied health professionals suggested to consult otolaryngologist and $43 \%$ preferred to refer their patient to audiologist in case of hearing loss as represented in Fig. 5. In case on ringing sensation in ear, $54 \%$ of the allied health professionals suggested to consult an otolaryngologist. Most of the allied health professionals were knowing the adverse effect of noise induced hearing loss. $91 \%$ of the allied health professionals were aware about courses like "Speech and Hearing" and "Audiology and Speech Language Pathology". Only $60 \%$ of the allied health professionals agreed for the fact that vertigo can occur with tinnitus. $78 \%$ of the allied health professionals were aware of the fact that balance problem could be due to dysfunction in inner ear.

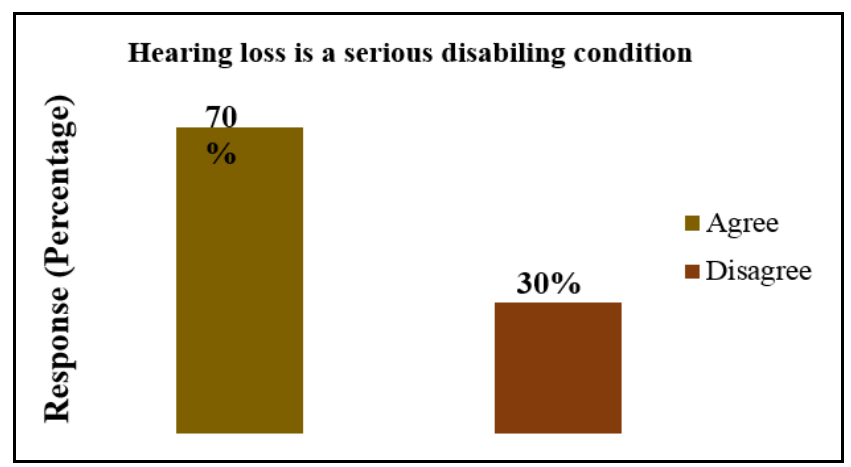

Fig. 1: Responses obtained for the question asked regarding hearing loss as serious health condition 


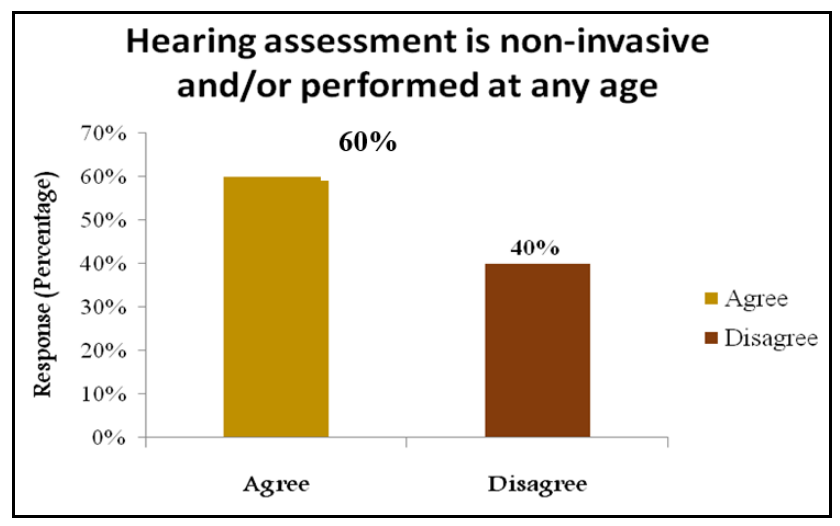

Fig. 2: Responses obtained for the question been asked regarding hearing assessment as an invasive/non-invasive procedure.

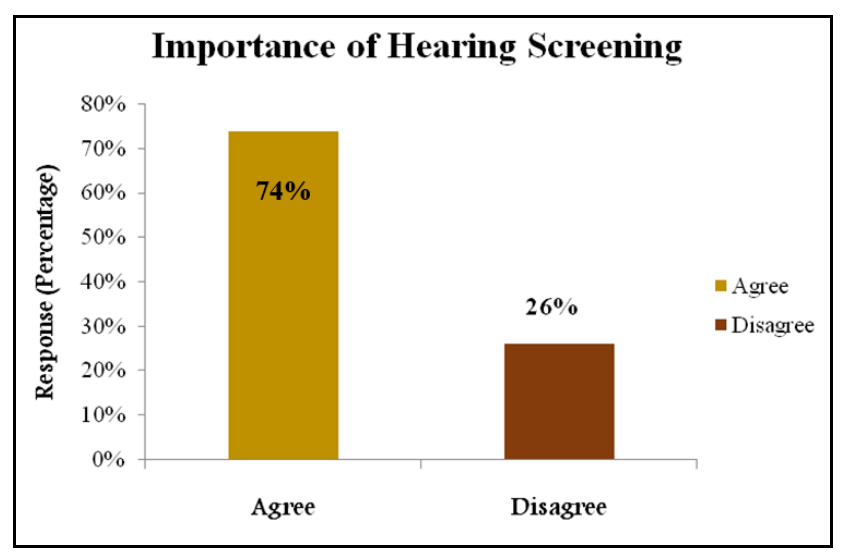

Fig. 3: Responses obtained for the question asked regarding importance of hearing screening

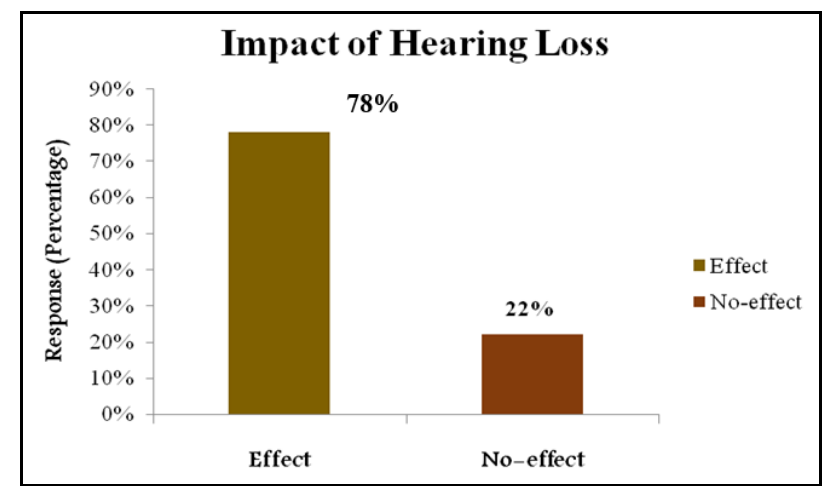

Fig. 4: Responses obtained for the question asked to assess regarding the consequences of hearing loss in hearing impaired individuals

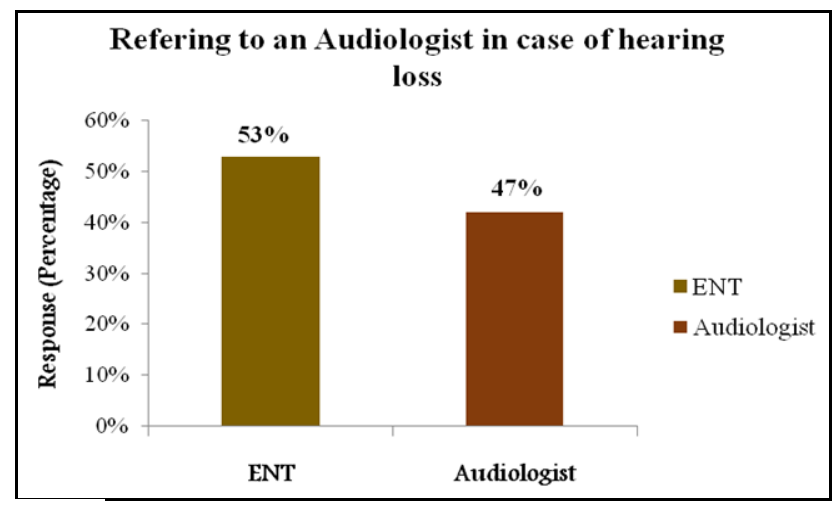

Fig. 5: Responses obtained for the question asked being asked regarding appropriate referral for an individual with hearing loss

\section{Discussion}

Any kind of health-related venture implemented can function successfully, depending upon the knowledge and attitude of the professionals involved. This study illustrates that in spite of having adequate knowledge about speech and hearing field, there is a dearth of knowledge among allied health professional regarding the role of an audiologist in treating people with hearing loss. As the result depicts that only $53 \%$ of the allied health professional agree to the notion that hearing-impaired individuals must be sent to an audiologist for further hearing related assessment. Similar findings was found in a study conducted by Brown et al. $(2006)^{12}$ wherein they found that only $50 \%$ of the allied health professional were aware about referral to an ENT physician/pediatric audiologist regarding hearing loss. Another study done by Olusanya and Roberts $2006,{ }^{13}$ concluded that medical students demonstrated greater awareness of early detection regarding infant hearing loss as compared to other allied health physicians, however both the groups exhibited lack of awareness and knowledge with respect risk factors in case of pediatric hearing loss. ${ }^{13}$ According to a survey done on hearing loss prevention in Mexico, $2009^{11}$ it was revealed as there is lack of knowledge and supportive attitude among general practitioners which resulted significant delay in identifying hearing loss individuals, diagnosing and providing adequate intervention. For the question on the importance of hearing screening and the need for spreading awareness with respect to speech and hearing, most of the participants showed a positive attitude, higher number of allied health professionals showed better attitude as compared to medical students.

According to the result obtained in the present study, more than $50 \%$ of the allied health professionals participants responded as strongly agree or agree that children with hearing loss can be fitted with hearing aids and should be given speech therapy. Another study done on allied health professionals working in Mexico ${ }^{11}$ showed similar kind of result wherein $40 \%$ responded to agree or strongly agree, whereas almost $32 \%$ responded as disagree or strongly disagree. Result obtained strongly highlights the importance 
of auditory training and speech therapy as an essential aspect in the overall treatment of children with hearing loss.

As physicians from any specialty play a vital role, hence it is very crucial for them to possess adequate knowledge regarding the noninvasiveness and age-related characteristics of hearing tests, so that they can provide proper guidance to the families for appropriate assessment and adequate intervention options. ${ }^{14}$ More than $50 \%$ of the participants responded correctly when they were asked regarding the nature of hearing tests whether invasive or not and can these tests be administered at any age. This is really remarkable as it is more in number than the response obtained in the previous study conducted in Mexico ${ }^{11}$ wherein the researchers had revealed that only $45.7 \%$ of the physicians were able to perform correctly regarding the nature of hearing tests are noninvasive and agreed that it can be performed at any age.

\section{Conclusion}

It is very essential for every health related professional to have adequate knowledge regarding individuals with vestibular and hearing problem. Programs being framed to implement focusing on early identification and intervention with respect to hearing loss can be effective depending on various factors, and awareness, ample knowledge among the medical professionals has been an considered to be an important factor in this process. Current study showed that even though allied health professionals showed adequate knowledge and positive attitude towards hearing impairment and vestibular disorders, but still there are certain areas which needs attention and concern to develop awareness among these professionals. There is a need to educate these professionals regarding role of audiologists and speech language pathologist in hearing and balance disorders. Role of amplification device in management of individuals with hearing impairment need to be focused, while educating these professionals. In order to conclude, though the participants demonstrated an overall good knowledge and attitude towards the basic areas related to hearing loss. However, there are gaps in certain areas where there is a need to increase awareness and educate them regarding the role of an audiologist/speech language pathologist.

\section{Conflict of Interest: Nil.}

\section{References}

1. "What is Allied Health?". Association of Schools of Allied Health Professionals. Retrieved 6 March 2014.
2. Varshney S. Deafness in India. Indian J Otol 2016;22:73.

3. White KR, Forsman I, Eichwald J, Munoz K. The evolution of early hearing detection and intervention programs in the United States. In Seminars in Perinatology 2010;34:170-9.

4. Yoshinaga- Itano C. Early intervention after universal neonatal hearing screening: impact on outcomes. Dev Disabilities Res Rev 2003;9:252-66

5. Yoshinaga-Itano C. Levels of evidence: Universal newborn hearing screening (UNHS) and early hearing detection and intervention systems (EHDI). J Commun Disord 2004;37:45165 .

6. Kennedy C, McCann D, Campbell MJ, Kimm L, Thornton R. Universal newborn screening for permanent childhood hearing impairment: An 8-year follow-up of a controlled trial. Lancet 2005;366:660-2.

7. Goedert MH, Moeller MP, White KR. Midwives' knowledge, attitudes, and practices related to newborn hearing screening. $J$ Midwifery Women's Health 2011;56:147-53.

8. Campos AC, Shirane HY, Takemoto PV, Lourenço EA. Universal newborn hearing screening: knowledge of pediatricians and neonatologists in the city of Jundiaí, São Paulo, Brazil. Brazilian journal of otorhinolaryngology. 2014;80:379-85.

9. Yerraguntla K, Ravi R, Gore S. Knowledge and attitude of pediatric hearing impairment among general physicians and medical interns in coastal Karnataka, India. Indian J Otol $2016 ; 22: 183$.

10. R. Ravi, D.R. Gunjawate, K. Yerraguntla, L.E. Lewis, B. Rajashekhar A national survey of knowledge, attitude and practices among pediatricians towards newborn hearing screening in India. Int J Pediatr Otorhinolaryngol 2017;95:914.

11. López-Vázquez M, Berruecos P, Lopez LE, Cacho J. Attitude and knowledge of hearing loss among medical doctors selected to initiate a residency in Mexico. Int J Audiol 2009;48:101-7.

12. Brown NC, James K, Liu J, Hatcher PA, Li Y. Newborn hearing screening. An assessment of knowledge, attitudes, and practice among Minnesota physicians. Minn Med 2006;89:50-4.

13. Olusanya BO, Roberts AA. Physician education on infant hearing loss in a developing country. Dev Neurorehabil 2006;9:373-7.

14. Dorros C, Kurtzer-White E, Ahlgren M, Simon P, Vohr B. Medical home for children with hearing loss: physician perspectives and practices. Pediatr 2007;120:288-94.

How to cite this article: Sanju HK, Aggarwal K, Yadav AK. Knowledge and attitude toward hearing impairment and vestibular disorders among allied health professionals in Delhi NCR. Indian J Anat Surg Head Neck Brain 2019;5(2):44-7. 